\title{
58-jähriger Patient mit Potenzabnahme und Leistungsschwäche
}

? Zu Ihnen kommt ein 58-jähriger Patient, der über eine Abnahme der Potenz und der Libido sowie eine allgemeine Leistungsschwäche klagt. Woran denken Sie?

Antwort Ganz allgemein an eine internistische Erkrankung, insbesondere natürlich kardiovaskuläre Erkrankungen, im Besonderen sollte aber auch immer ein Testosteronmangel in Erwägung gezogen werden.

Kommentar Testosteronmangel: Unter Umständen unspezifische Symptomatik.

Wie können Sie den Testosteronmangel feststellen?

Antwort Durch Messung des Serumtestosterons.

Kommentar Serumtestosteron:

- Normal: > $12 \mathrm{nmol} / \mathrm{l}$

- Grenzbereich: 8 - $12 \mathrm{nmol} / \mathrm{l}$

- Testerosteronmangel: $<8 \mathrm{nmol} / \mathrm{l}$

? Gibt es Regeln, zu welcher Tageszeit Sie den Testosteronspiegel messen sollten?

Antwort Ja, morgens.

Kommentar Der abendliche Testosteronwert liegt 20 - $30 \%$ unter dem morgendlichen.

\section{Merke}

Testosteron sollte morgens gemessen werden.

? Zu welchen Symptomen führt denn ein Testosteronmangel?

Antwort Zu Störungen der sexuellen Funktion, der Knochenmineralisation, des Muskel- und Fettstoffwechsels, des Blutes sowie zu Allgemeinsymptomen.
Kommentar Mögliche Symptomatik bei Testosteronmangel:

- Sexualfunktion:

- Libidoverminderung

- Potenzstörungen

- Knochenmineralisation:

- Osteoporose

- Muskel- und Fettstoffwechsel:

- Muskelatrophie

- Muskelschwäche

- Zunahme des Körperfettgewebes

- Blut:

- Anämie

- Allgemeinbeschwerden:

- Leistungsminderung

- Depression

- Müdigkeit

?

Welches sind überhaupt die wichtigsten Ursachen einer Osteoporose bei einem Mann?

Antwort Alkoholabusus, Einnahme von Glukokortikoiden, Hypogonadismus.

Kommentar Osteoporose beim Mann: Immer auch konkurrierende Ursachen zum Testosteronmangel abklären.

\section{Merke}

Hauptursache der Osteoporose beim Mann $\rightarrow$ Alkoholabusus und Steroideinnahme.

\section{?}

Kennen Sie den Unterschied zwischen primärem und sekundärem Hypogonadismus?

Antwort Beim primären Hypogonadismus liegt die Störung im Hoden, beim sekundären im Bereich der Hypophyse oder des Hypothalamus.

Kommentar Differenzierung primärer sekundärer Hypogonadismus:
- Primärer Hypogonadismus:

- verminderte Testosteronproduktion im Hoden mit erhöhten Gonadotropinspiegeln (hypergonadotroper Hypogonadismus)

- Sekundärer Hypogonadismus:

- verminderte Sekretion von Gonadotropin-Releasing-Hormon (hypogonadotroper Hypogonadismus)

? Wodurch ist das Testosterondefizit beim älter werdenden Mann bedingt?

Antwort Durch einen sekundären Hypogonadismus.

Kommentar Beim älter werdenden Mann ist der Testosteronmangel eine Folge der nachlassenden hypothalamischen Sekretion von Gonadotropin-Releasing-Hormon.

? Wie würden Sie bei dem vorhin erwähnten 58-jährigen Patienten jetzt weitermachen?

Antwort Sorgfältige Anamneseerhebung im Hinblick auf mögliche Symptome eines Testosteronmangels, Messung des Testosteronspiegels.

Kommentar Über die Diagnosestellung und die therapeutische Konsequenz eines symptomatischen Testeronmangels muss im Einzelfall entschieden werden. Je niedriger der Testosteronspiegel und je stärker die potenziellen Symptome des Testosteronmangels, desto eher sollte eine Testosteronsubstitution erfolgen.

? Wer übernimmt eigentlich die Kosten für eine Testosteronsubstitution?

Antwort Die Krankenkassen.

Kommentar Bei Vorliegen eines Hypogonadismus werden die Kosten für eine Testosteronbehandlung derzeit noch von den Kassen übernommen. 
Wie führen Sie die

Substitutionsbehandlung durch?

Antwort Testosteron kann auf unterschiedliche Weise zugeführt werden: p. o., i. m. oder transdermal.

Kommentar Testosteronsubstitution:

- Per os:

- Testosteron-Unedecanoat (Andriol): 2-4-mal $40 \mathrm{mg} / \mathrm{d}$

- Intramuskulär:

- Testosteron-Enantat: 250 mg alle 2- 3 Wochen

- Testosteronundecanoat: $1000 \mathrm{mg}$ alle 10 - 14 Wochen

- Transdermal:

- 1 - 2 Pflaster à $2,5 \mathrm{mg} / \mathrm{d}$

- Gel

- Weitere Möglichkeiten:

- Implantat eines Hormondepots

- sublingual

? Das sind ja eine ganze Menge Möglichkeiten. Welche Vor- und Nachteile beachten Sie bei der Auswahl?

Antwort Die Kosten, die Praktikabilität der Anwendung, die Spiegelschwankungen.

Kommentar Vor- und Nachteile unterschiedlicher Testosteronapplikationsformen:

- Per os:

- hohe Kosten

- variable Resorption

- Anwendung mehrfach proTag

- Pflaster:

- Hautreizung

- hohe Kosten

- Intramuskulär:

- preiswert

- hohe Spitzenwerte

- Schwankungen

Welches ist die wichtigste Maßnahme vor Einleitung einer Therapie?

Antwort Ausschluss eines Prostatakarzinoms.

\section{Cave}

Vor Testosteronsubstitution Ausschluss eines Prostatakarzinoms durch Tastbefund, transrektale Sonografie, PSA-Wert.
? Welche Kontrolluntersuchungen führen Sie während der Substitutionsbehandlung durch?

Antwort Zum einen Kontrolle der Wirkung auf die Symptomatik, die im Vordergrund stand. Dann Kontrolluntersuchung im Hinblick auf mögliche Nebenwirkungen, insbesondere auf die wachstumsfördernde Wirkung bei bestehendem Prostatakarzinom.

Kommentar Unter Testosteronbehandlung sind regelmäßige Kontrolluntersuchungen notwendig.

? Wie sehen die Kontrolluntersuchungen genau aus?

Antwort Anamnese: Wirkung auf Sexualität und Leistungsfähigkeit, körperliche Untersuchung: insbesondere Muskel- und Fettverteilung, Laboruntersuchungen: hier insbesondere Blutbild und Lipide, Untersuchung im Hinblick auf die Prostata.

Kommentar Kontrolluntersuchungen bei Testosteronsubstitution:

- Anamnese:

- Wirkung auf die subjektiven Symptome des Testosteronmangels: Leistungsfähigkeit, Sexualität, Befindlichkeit

- Körperliche Untersuchung:

- Muskelmasse, Muskelfett, Behaarung

- Laborwerte:

- Blutbild, Lipide, Testosteron im Serum

- Prostata:

- Tastbefund, sonografischer Befund, PSA-Wert

- Knochen:

- Knochendichtemessung

Sie behandeln einen Patienten mit etwas erniedrigtem Testosteronspiegel. Bei ihm stehen die Potenzstörungen im Vordergrund. Lässt sich durch Testosteron die Potenz steigern?

Antwort Schlecht.

\section{Merke}

Testosteronwirkung auf die Sexualfunktion $\rightarrow$ Die Libido lässt sich besser steigern als die Potenz.

Welche anderen Ursachen von

Potenzstörungen beim älter

werdenden Mann berücksichtigen Sie?

Antwort Gleichzeitig bestehende internistische Erkrankungen, insbesondere kardiovaskuläre Erkrankungen, Diabetes mellitus, chronische Niereninsuffizienz, außerdem Medikamente, z. B. Betablocker, sowie psychosomatische Faktoren.

Kommentar Der Hypogonadismus ist nur eine mögliche Ursache neben zahlreichen anderen für die männliche Impotenz.

Wann setzen eigentlich die „Wechseljahre“ des Mannes ein?

Antwort Überhaupt nicht.

Kommentar Es kommt ab dem 40. Lebensjahr zu einem langsamen Absinken des freien Testosterons, nicht jedoch - wie bei der Frau - zu einem abrupten Abfall.

Gibt es eigentlich unterschiedliche Normwerte für Testosteronspiegel bei alten Männern und jungen Männern?

Antwort Nein.

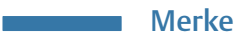

Im Hinblick auf die Substitution werden für alte und junge Männer gleiche Normwerte angesetzt.

Adaptiert nach: Berthold Block, Facharztprüfung Innere Medizin, 3000 kommentierte Prüfungsfragen. 5., vollständig überarbeitete Auflage 2017

ISBN 9783131359551 